\title{
Tortugas Charapa un aporte para el turismo comunitario y conservación de la biodiversidad
}

\section{Charapa Turtles a contribution to community tourism and biodiversity conservation}

Carlos Mestanza Ramón. ${ }^{1}$, Marco Antonio Toledo Villacís. ${ }^{2}$ \& Angel Edberto Cunalata García. ${ }^{3}$

\begin{abstract}
In the last decade in the Republic of Ecuador tourism has been strengthened considerably and one of the beneficiaries has been the indigenous and rural communities through community-based tourism. This type of tourism has not achieved the goals for which it was conceived. The present study aims to demonstrate the problem of community-based tourism in a community in Yasuni National Park focusing on legal and economic aspects and the implementation of a solution through a proposal of a project linking academia, government institutions and communities, focusing on the use of a threatened species and taking advantage of its conservation program through tourism use. The methodology is based on a bibliographic compilation and the application of professional experiences and fieldwork with the use of interviews and observation. The main results indicate that there are serious problems in legal aspects and economic management; and, that the best option for implementing a conservation program is the Charapa species (Podocnemis unifilis and Podocnemis expansa). Managers are motivated to carry out training and strengthening programs that really focus on the weaknesses of the communities that carry out this type of tourism.
\end{abstract}

Keywords: Ecuador, community tourism, Amazon, economy, Podocnemis unifilis.

\section{Resumen}

En la última década en la República del Ecuador se fortaleció notablemente el turismo y uno de los beneficiados fueron las comunidades indígenas y rurales mediante el turismo comunitario. Este tipo de turismo no ha logrado alcanzar las metas por las que

\footnotetext{
${ }^{1}$ Escuela Superior Politécnica de Chimborazo sede Orellana, carlosmestanza123@hotmail.com

${ }^{2}$ Escuela Superior Politécnica de Chimborazo sede Orellana, marcotoledov1977@gmail.com

${ }^{3}$ Escuela Superior Politécnica de Chimborazo sede Orellana, angeledberto@yahoo.com
} 
se concibió. El presente estudio pretende demostrar la problemática del turismo comunitario en una comunidad en el Parque Nacional Yasuni enfocándose en aspectos legales y económicos y la implementación de una solución mediante una propuesta de un proyecto de vinculación entre la academia, instituciones gubernamentales y comunidades, centrándose en el uso de una especie amenazada y aprovechar su programa de conservación mediante un uso turístico. La metodología se basa en una recopilación bibliográfica y la aplicación de experiencias profesionales y trabajo de campo con el uso de entrevistas y observación. Los principales resultados indican que existen graves problemas en aspectos legales y el manejo económico; y, que la mejor opción para implementar un programa de conservación es la especie Charapa (Podocnemis unifilis y Podocnemis expansa). Se motiva a los gestores realizar programas de capacitación y fortalecimiento enfocándose realmente en las debilidades de las comunidades que realizan este tipo de turismo.

Palabras claves: Ecuador, Turismo Comunitario, Amazonía, Economía, Podocnemis unifilis.

\section{Introducción}

El turismo se considera un método eficaz para reducir la pobreza en algunas comunidades indígenas y rurales (Croes, 2014; Leo, 2019), proporciona empleos en sus diversas actividades, así como oportunidades para vender productos locales (Carter, Schmidt, \& Hirons, 2015; Lee, 2013). El turismo basado en la comunidad ha sido ampliamente identificado por su capacidad para mejorar las economías locales, y se ha introducido en diversos países (Dodds, Ali, \& Galaski, 2018).

El uso de los recursos naturales en áreas protegidas ha dado lugar a conflictos sociales. Tales conflictos de conservación o conflictos socioambientales pueden definirse como situaciones que ocurren cuando dos o más partes tienen intereses, percepciones, opiniones o emociones opuestas sobre un objetivo dado (Carlos Mestanza et al., 2019; Zoppi, 2018). Esta definición reconoce que pueden ocurrir conflictos entre seres humanos y que, en el contexto de áreas protegidas, pueden surgir conflictos cuando los intereses de conservación de una de las partes se ven amenazados por la posición de aquellos con una visión diferente (Mestanza-Ramon, Cunalata-García, Jiménez-Gutiérrez, \& Chacha-Bolaños, 2019).

En los últimos tiempos, las áreas naturales protegidas han sufrido en gran medida las influencias antropogénicas (C. Mestanza et al., 2019), y por esta razón es necesario implementar nuevas técnicas de conservación para las áreas protegidas (Mestanza-Ramón, Sánchez Capa, Figueroa Saavedra, \& Rojas Paredes, 2019). La superficie de las áreas protegidas en todo el mundo en los últimos años ha aumentado hasta el punto de que actualmente el 14\% de la superficie terrestre tiene alguna forma de protección. La legislación en un área protegida puede generar conflictos perjudiciales cuando no se gestiona correctamente (C. Mestanza, Saavedra, et al., 2018). Los diversos actores gubernamentales 
y la población local pueden sentirse afectados negativamente por el acceso y uso de los recursos naturales en las áreas protegidas (Mooser, Anfuso, Mestanza, \& Williams, 2018). Los conflictos mal administrados en las áreas protegidas a menudo pueden empeorar la situación de lo que comenzó (Mestanza-Ramón et al., 2019).

Durante más de un siglo, la designación y gestión de áreas protegidas se ha realizado con el objetivo de permitir el uso actual de la biodiversidad, generalmente a través del turismo, al tiempo que se preservan los recursos para las generaciones futuras (Chung, Dietz, \& Liu, 2018). Las relaciones entre la conservación de la biodiversidad y los servicios del ecosistema son ampliamente debatidas (C. Mestanza, Piccardi, \& Pranzini, 2018). Sin embargo, todavía no está claro cómo la conservación de la biodiversidad y sus servicios interactúan con diferentes estrategias en y alrededor de las áreas protegidas (Beissinger, Ackerly, Doremus, \& Machlis, 2017). Uno de los principales objetivos para la conservación en áreas protegidas enfatiza la protección de los sistemas naturales y la biodiversidad (Ramon et al., 2019; Zarębski, Kwiatkowski, Malchrowicz-Mośko, \& Oklevik, 2019). El otro enfatiza la contribución de los servicios del ecosistema al bienestar humano. Por lo tanto, comprender la relación entre los servicios ecosistémicos de áreas protegidas y la biodiversidad es un desafío importante para la ciencia de la sostenibilidad (Graves, Pearson, \& Turner, 2017).

Podocnemis unifilis (tortuga charapa) es la especie de tortuga de agua dulce más común en la Amazonía de América del Sur. Las presiones antrópicas como caza impulsadas por el consumo de carne y huevos, la destrucción de nidos y la degradación del hábitat en los asentamientos humanos a la orilla del río han llevado a la disminución de la población de Podocnemis unifilis (Hirano et al., 2019) y la reclasificación de esta especie como vulnerable en la Lista Roja de Especies Amenazadas de la UICN. Podocnemis unifilis anida en julio y agosto, con un período de incubación que oscila entre 45 y 97 días (Fachín Terán \& Von Mülhen, 2003). El uso de la especie como fuente de alimento es el principal factor que contribuye a la disminución de las poblaciones (Caputo, Canestrelli, \& Boitani, 2005). La madurez sexual retrasada y la larga vida reproductiva son características de la demografía de tortugas que hacen que sus poblaciones sean especialmente sensibles a la explotación de juveniles y adultos (Alves \& Santana, 2008; Noronha et al., 2016); y, la recolección de huevos y crías, que naturalmente mantienen altas tasas de mortalidad (Espinoza, Mertins, Gama, Fernandes Patta, \& Mathews, 2017). Es evidente que aumentar la tasa de supervivencia de las primeras etapas de la vida no es eficaz para la recuperación de la población cuando las tasas de mortalidad de los juveniles y adultos están aumentando (Conway-Gómez, Reibel, \& Mihiar, 2014). Sin embargo, la gestión de las primeras etapas de la vida podría ser una opción viable cuando el objetivo es un programa de explotación sostenible de la población. La protección del sitio de anidación o los programas de recolección controlada de huevos, por ejemplo, podrían ayudar a aumentar la tasa de supervivencia de los adultos, especialmente cuando su colecta se desarrolla durante el período de desove. 
El presente estudio analiza la realidad del turismo comunitario en la comunidad Río Indillama en el Parque Nacional Yasuní y diseña un proyecto de vinculación interactuando la academia, instituciones gubernamentales y comunidades locales. El análisis y diseño se realiza mediante revisión bibliográfica y la descripción de experiencias de campo, permitiendo implantar una nueva idea de turismo comunitario basado en la conservación que permita dinamizar la economía local y fortalezca la conservación de la biodiversidad.

\section{Metodologia}

Área de estudio

La comunidad Río Indillama se localiza en el límite norte del Parque Nacional Yasuní (PNY), a 80 kilómetros en la ruta El Coca - Nuevo Rocafuerte margen derecho. Su territorio abarca una extensión de 14.400 hectáreas. Para acceder a la zona de estudio desde la ciudad de El Coca se dispone de dos opciones, vía fluvial y una mixta terrestre y fluvial. El PNY se encuentra en la cuenca amazónica del noreste de Ecuador, con la mayor proporción en la provincia de Orellana su altitud es variante entre 200 y $300 \mathrm{msnm}$. El clima se caracteriza por una precipitación anual promedio de $3000-3600 \mathrm{~mm}$ y temperaturas anuales promedio de $24^{\circ} \mathrm{C}$ con una estación lluviosa prolongada y fluctuaciones estacionales no marcadas en la precipitación y la temperatura (Bass et al., 2010). El parque es conocido por su extraordinaria biodiversidad un recurso con gran potencial para realizar diversas actividades y explorar productos turísticos.

\section{Metodología}

La metodología de la presente investigación busca analizar las realidades del turismo comunitario en la Comunidad del Río Indillama, Parque Nacional Yasuní, Ecuador. El estudio utiliza una metodología mixta (cuantitativa cualitativa), no experimental y transversal, se realizó una revisión bibliográfica y de campo (entrevistas y encuestas). El trabajo de campo se realizó entre enero y noviembre de 2019.

Para el análisis de la realidad del turismo comunitario el estudio se centró en dos aspectos fundamentales el legal y el económico. Para el análisis legal se recabó documentación que permita describir su estado referente a constitución y funcionamiento del emprendimiento turístico. Mientras que para describir la realidad económica se recabaron los libros y apuntes contables, los cuales permitan describir si la actividad realmente está dinamizando la economía de la comunidad.

Para la implementación de la propuesta de diseño para una transformación o valor agregado al turismo comunitario se utilizó la experiencia profesional de los investigadores los cuales aportaron diversas ideas y estrategias. La investigación también se fortaleció con información importante recabada mediante entrevistas a diversos actores locales y turistas en general. En la entrevista se utilizaron preguntas semiestructuradas permitiendo dar un margen de libertad a los actores y de esta forma poder receptar diversos puntos de vista que aporten con el fortalecimiento del diseño. Para el planteamiento del proyecto se pretende utilizar una especie 
amenazada, para identificar su problemática y trabajar en la recuperación de su población y en su proceso sea aprovechada como un producto turístico de conservación sostenible.

\section{Resultados}

Para dar respuesta a los objetivos de la presente investigación, los resultados se presentan en dos apartados, el primero que narra la realidad del turismo comunitario en la Comunidad Rio Indillama desde dos aspectos el legal y económico; y, finalmente se plantea un diseño de proyecto de vinculación que permita la transformación del turismo comunitario mediante la implementación de un valor agregado:

\section{Realidad del turismo comunitario.}

En el ámbito legal se ha logrado identificar que no existen políticas y reglamentos para la operación del emprendimiento turístico. Esto ha ocasionado un desorden en el funcionamiento y que reiteradamente los administradores de turno pierdan el norte para lo cual se concibieron; y, no se puede tener un norte cuando no existe una meta y objetivos establecidos. En cuanto al funcionamiento hasta el emprendimiento turístico no cuenta con todos los permisos que habiliten su funcionamiento como personería jurídica, patente, licencias ambientales y permisos de bomberos.

En lo económico el emprendimiento de turismo comunitario en la Comunidad Río Indillama no dispone de libros contables o apuntes en general que permitan realizar un análisis documental sobre su situación actual y su avance en el tiempo. Para poder describir fue necesario basarse en entrevistas y percepción antrópica local. El turismo comunitario poco o nada ha contribuido a la economía y a la mejora de la calidad de vida, en la comunidad Río Indillama. Los comuneros se sienten decepcionados con los administradores del emprendimiento. Los pocos ingresos y la mala distribución de las regalías que deja el turismo han provocado conflictos sociales. Actualmente, el problema de las bajas o nulas regalías económicas ha sido un factor muy fuerte para que los miembros de la comunidad muestren una falta de interés en la vinculación y el trabajo en equipo. La implementación de la infraestructura ha sido posible gracias a los aportes de los gobiernos locales y organizaciones, en la actualidad la infraestructura presenta deterioros que no pueden ser reparados por la falta de un manejo adecuado de los pocos recursos que ingresan. No hay claridad en el manejo de los recursos económicos, la falta o inexistencia de tarifas por los escasos servicios que ofrecen contribuyen aún más a la profunda crisis en el manejo y gestión del turismo comunitario. En la comunidad hay una crisis en el modelo de gestión. No tienen un plan estratégico que permita el buen funcionamiento de la empresa independiente de los jefes de turno.

\section{Proyecto de Vinculación.}

La Escuela Superior Politécnica de Chimborazo (ESPOCH) luego del análisis de la realidad en los aspectos legales y económicos; y, para atender a la solicitud de los representantes de la comunidad decide emprender un proyecto de vinculación con la sociedad y de esta forma dar solución a la problemática en el turismo comunitario. 
El proyecto de vinculación pretende i) diseñar un plan estratégico dinamizador de la economía mediante la implementación de un producto turístico de conservación sostenible, para mejorar la calidad de vida y protección del ambiente en la comunidad Río Indillama, Parque Nacional Yasuní, ii) describir la ecología de la Charapa (Podocnemis unifilis \& Podocnemis expansa) en el proceso de reproducción para su aprovechamiento turístico, iii) Implementar cultivos orgánicos de especies vegetales utilizando técnicas locales para aprovechamiento en la nutrición de la Charapa (Podocnemis unifilis \& Podocnemis expansa) y iv) socializar con los actores sociales locales y personas en general interesadas sobre la implementación de nuevas estrategias de turismo comunitario de conservación. Se obtendrá un plan estratégico de mejora con la implementación de un producto turístico de conservación mediante el uso de una de sus especies representativas la tortuga Charapa (Podocnemis unifilis \& Podocnemis expansa) en donde el turista pueda pagar por realizar actividades que se establecerán en los procesos de reproducción (recolección de huevos, nidificación, eclosión, manejo de neonatos y liberación de especie al medio natural). Surgiendo una nueva idea un turismo comunitario de conservación en donde se aprovechen las etapas de reproducción de la especie para crear un turismo estacional el cual permita abrirse nuevos espacios y atraer a un grupo de turistas selecto en el campo de la conservación.

El proyecto beneficiará a la comunidad Río Indillama y a las comunidades asentadas en la zona de influencia del Parque Nacional Yasuní sector norte, de forma directa el proyecto beneficiará a 400 personas y de forma indirecta a más de 5000 personas. Los participantes del proyecto serán catedráticos y alumnos de la ESPOCH sede Orellana y personal de la comunidad Río Indillama. El proyecto tendrá una duración de 36 meses en el primer año se desarrollará $2 / 3$ de los objetivos planteados y para el según y tercer año se pretende entregar el producto turístico final elaborado mediante un manual de funcionamiento. Se pretende que este proyecto forme parte de un proyecto futuro para la conservación de especies en estado vulnerable por el deterioro de zonas prístinas.

\section{Discusión}

Al incorporar el turismo comunitario en los planes de vida de las comunidades, los líderes han encontrado una forma relativamente fácil de incluir, al menos en el papel, una buena parte de la comunidad. En otras palabras, estos proyectos son una herramienta comúnmente utilizada por los líderes para demostrar su manejo. Pero en la práctica, el nivel de compromiso, responsabilidad y participación de los miembros de la comunidad en los programas de turismo comunitario es muy variable y no siempre corresponde al patrón de distribución de los ingresos del programa. Esta inequidad es el resultado de la interacción de intereses contrapuestos que existe en todas las comunidades humanas y que sólo un liderazgo fuerte, sabio y honesto puede controlar.

La mala gestión del marketing y la falta de un producto bandera es uno de los principales problemas que se evidencia en la baja demanda del turismo. La comunidad requiere de un cambio en el modelo de gestión inmediato, que debe mostrar las debilidades y amenazas a 
través de un FODA, que debe ser complementado y acompañado por la implementación de un producto turístico estrella.

La profusión de proyectos de capacitación, diseño y evaluación de programas de turismo comunitario por parte de fundaciones y organizaciones afines es sorprendente si se la contrasta con la escasez de proyectos y actividades que efectivamente traigan turistas a los programas. El Ministerio de Turismo no escapa a esta tendencia, tres de los cuatro componentes de su programa de Turismo Comunitario, son la asistencia técnica, la capacitación y la implementación de estándares de calidad, sólo el último componente es la promoción. A nadie se le ocurriría negar la necesidad de capacitación, evaluación y desarrollo de estándares adecuados para el éxito de un programa de turismo. Sin embargo, muchas de las fundaciones y agencias de cooperación se quedan allí porque es una forma mucho más fácil de demostrar la gestión presentando un informe con los resultados de unos días de evaluación o un típico taller de capacitación de dudosos resultados, considerando las metodologías antipedagógicas modulares que utilizan.

\section{Conclusiones}

Es necesario aceptar que muchos de los programas de turismo comunitario propuestos no tienen los atributos y la fuerza necesaria para despegar, mantener y crecer. Esta aceptación debe significar la asignación de los fondos y esfuerzos que se les proporcionen para otros fines. También es tiempo de usar nuestra imaginación y originalidad para proponer otras alternativas de producción y desarrollo sostenible en las comunidades. Esa es una tarea de todos, pero de quienes tienen la responsabilidad última de la evaluación y la implementación de las mismas deben ser realizadas por miembros de las comunidades sin la influencia de organizaciones externas que, en algunos casos, se han beneficiado del mantenimiento del statu quo o han demostrado que no tienen la capacidad de cambiarlo.

Las comunidades tienen un gran desafío por delante y sólo podrán afrontarlo con éxito si se preocupan lo antes posible por mejorar sus sistemas educativos. Este es un proceso complejo y de largo plazo que no se basa en hacer los habituales talleres relámpago; su objetivo final debe ser enseñar lo que no nacen sabiendo: respeto, responsabilidad, compromiso y un profundo amor por lo que hacen.

El proyecto de vinculación dinamizará la economía mediante la implementación de un producto turístico de conservación sostenible, para mejorar la calidad de vida y protección del ambiente en la comunidad Río Indillama, Parque Nacional Yasuní. En este proceso se utilizará dos especies emblemáticas de la comunidad (Podocnemis unifilis \& Podocnemis expansa). Al finalizar el proyecto los turistas podrán disfrutar de la explicación y práctica de todo un proceso de conservación y fortalecimiento de la biodiversidad y su funcionamiento.

Como implicación para la gestión, los resultados de esta investigación pueden ser útiles, en primer lugar, para la comunidad local que se beneficia de la actividad generada por el turismo comunitario. Los gestores del turismo comunitario deben seguir apostando por el desarrollo 
sostenible del mismo, ya que podría generar numerosos beneficios para la comunidad local, así como mejorar la conservación de los recursos naturales. Por otro lado, las entidades gubernamentales deben considerar los resultados obtenidos en este estudio, ya que podrían mejorar el desarrollo turístico en áreas con potencial para el turismo comunitario, con el fin de promover el desarrollo sostenible de las comunidades locales.

\section{Referencias bibliográficas.}

Alves, R. R. N., \& Santana, G. G. (2008). Use and commercialization of Podocnemis expansa (Schweiger 1812) (Testudines: Podocnemididae) for medicinal purposes in two communities in North of Brazil. Journal of Ethnobiology and Ethnomedicine, 4(1), 3. https://doi.org/10.1186/1746-4269-4-3

Bass, M. S., Finer, M., Jenkins, C. N., Kreft, H., Cisneros-Heredia, D. F., McCracken, S. F., ... Villa, G. (2010). Global conservation significance of Ecuador's Yasuní National Park. PloS One, 5(1), e8767.

Beissinger, S. R., Ackerly, D. D., Doremus, H., \& Machlis, G. E. (2017). Science, conservation, and national parks. University of Chicago Press.

Caputo, F. P., Canestrelli, D., \& Boitani, L. (2005). Conserving the terecay (Podocnemis unifilis, Testudines: Pelomedusidae) through a community-based sustainable harvest of its eggs. Biological Conservation, 126(1), 84-92. https://doi.org/https://doi.org/10.1016/j.biocon.2005.05.004

Carter, N. H., Schmidt, W. S., \& Hirons, C. A. (2015). An International Assessment of Mangrove Management: Incorporation in Integrated Coastal Zone Management. Diversity, Vol. 7. https://doi.org/10.3390/d7020074

Chung, M. G., Dietz, T., \& Liu, J. (2018). Global relationships between biodiversity and naturebased tourism in protected areas. Ecosystem Services, 34, 11-23. https://doi.org/https://doi.org/10.1016/j.ecoser.2018.09.004

Conway-Gómez, K., Reibel, M., \& Mihiar, C. (2014). A predictive model of yellow spotted river turtle (Podocnemis unifilis) encounter rates at basking sites in lowland eastern Bolivia. Applied Geography, 53, 332-340. https://doi.org/https://doi.org/10.1016/j.apgeog.2014.06.026

Croes, R. (2014). The role of tourism in poverty reduction: an empirical assessment. Tourism Economics, 20(2), 207-226.

Dodds, R., Ali, A., \& Galaski, K. (2018). Mobilizing knowledge: Determining key elements for success and pitfalls in developing community-based tourism. Current Issues in Tourism, 2l(13), 1547-1568.

Espinoza, L. L., Mertins, O., Gama, G. S., Fernandes Patta, A. C. M., \& Mathews, P. D. (2017). A 
new Myxidium species (Myxozoa: Myxosporea) infecting the gallbladder of the turtle Podocnemis unifilis (Testudines: Podocnemididae) from Peruvian Amazon. Acta Tropica, 172, 75-79. https://doi.org/https://doi.org/10.1016/j.actatropica.2017.04.021

Fachín Terán, A., \& Von Mülhen, E. M. (2003). Reproducción de la taricaya podocnemis unifilis troschel 1848 (testudines: podocnemididae) en la várzea del medio solimões, Amazonas, Brasil. Ecología Aplicada, 2(1), 125-132.

Graves, R. A., Pearson, S. M., \& Turner, M. G. (2017). Species richness alone does not predict cultural ecosystem service value. Proceedings of the National Academy of Sciences, 114(14), 3774-3779.

Hirano, L. Q. L., Alves, L. da S., Menezes-Reis, L. T., Mendonça, J. dos S., Simões, K., Santos, A. L. Q., \& Vieira, L. G. (2019). Effects of egg exposure to atrazine and/or glyphosate on bone development in Podocnemis unifilis (Testudines, Podocnemididae). Ecotoxicology and Environmental Safety, 182, 109400. https://doi.org/https://doi.org/10.1016/j.ecoenv.2019.109400

Lee, T. H. (2013). Influence analysis of community resident support for sustainable tourism development. Tourism Management, 34, 37-46.

Leo, S. (2019). Pitfalls of tourism graduate students in presenting the ingredients of research proposals. Journal of Hospitality, Leisure, Sport \& Tourism Education, 24, 178-189. https://doi.org/https://doi.org/10.1016/j.jhlste.2019.03.003

Mestanza-Ramon, C., Cunalata-García, Á. E., Jiménez-Gutiérrez, M. Y., \& Chacha-Bolaños, A. N. (2019). Disposición a pagar por el ingreso a zonas de uso público en el Parque Turístico "Nueva Loja”, Sucumbíos-Ecuador. Polo Del Conocimiento, 4(2), 67-82.

Mestanza-Ramón, C., Sanchez Capa, M., Figueroa Saavedra, H., \& Rojas Paredes, J. (2019). Integrated Coastal Zone Management in Continental Ecuador and Galapagos Islands: Challenges and Opportunities in a Changing Tourism and Economic Context. Sustainability, Vol. 11. https://doi.org/10.3390/su11226386

Mestanza, C., Botero, C. M., Anfuso, G., Chica-Ruiz, J. A., Pranzini, E., \& Mooser, A. (2019). Beach litter in Ecuador and the Galapagos islands: A baseline to enhance environmental conservation and sustainable beach tourism. Marine Pollution Bulletin. https://doi.org/10.1016/j.marpolbul.2019.02.003

Mestanza, C., Piccardi, M., \& Pranzini, E. (2018). Coastal erosion management at Callao (Peru) in the 17th and 18th centuries: The first groin field in South America? Water (Switzerland), 10(7). https://doi.org/10.3390/w10070891

Mestanza, C., Saavedra, H. F., Gaibor, I. D., Zaquinaula, M. A., Váscones, R. L., \& Pacheco, O. M. (2018). Conflict and impacts generated by the filming of Discovery Channel's reality series "Naked and Afraid" in the Amazon: A Special case in the Cuyabeno Wildlife Reserve, 
Ecuador. Sustainability (Switzerland), 11(1). https://doi.org/10.3390/su11010050

Mestanza, Carlos, Saavedra, H. F., Gaibor, I. D., Zaquinaula, M. A., Váscones, R. L., \& Pacheco, O. M. (2019). Conflict and Impacts Generated by the Filming of Discovery Channel's Reality Series "Naked and Afraid" in the Amazon: A Special Case in the Cuyabeno Wildlife Reserve, Ecuador. Sustainability, 11(1), 50.

Mooser, A., Anfuso, G., Mestanza, C., \& Williams, A. T. (2018). Management implications for the most attractive scenic sites along the Andalusia coast (SW Spain). Sustainability (Switzerland), 10(5). https://doi.org/10.3390/su10051328

Noronha, R. C. R., Barros, L. M. R., Araújo, R. E. F., Marques, D. F., Nagamachi, C. Y., Martins, C., \& Pieczarka, J. C. (2016). New insights of karyoevolution in the Amazonian turtles Podocnemis expansa and Podocnemis unifilis (Testudines, Podocnemidae). Molecular Cytogenetics, 9(1), 73. https://doi.org/10.1186/s13039-016-0281-5

Ramon, C. M., Capa, M. S., Garcia, A. C., Gutierrez, M. J., Villacís, M. T., \& Velasco, A. A. (2019). Community Tourism In Ecuador: A Special Case In The Rio Indillama Community, Yasuní National Park. International Journal of Engineering Research \& Technology (IJERT), 08(06), 5 .

Zarębski, P., Kwiatkowski, G., Malchrowicz-Mośko, E., \& Oklevik, O. (2019). Tourism Investment Gaps in Poland. Sustainability, Vol. 11. https://doi.org/10.3390/su11226188

Zoppi, C. (2018). Integration of Conservation Measures Concerning Natura 2000 Sites into Marine Protected Areas Regulations: A Study Related to Sardinia. Sustainability, Vol. 10. https://doi.org/10.3390/su10103460 


\section{PARA CITAR EL ARTÍCULO INDEXADO.}

Mestanza Ramón, C., Toledo Villacís, M. A., \& Cunalata García, A. E. (2020). Tortugas Charapa un aporte para el turismo comunitario y conservación de la biodiversidad. Explorador Digital, 4(1), 55-65. https://doi.org/10.33262/exploradordigital.v4i1.1075

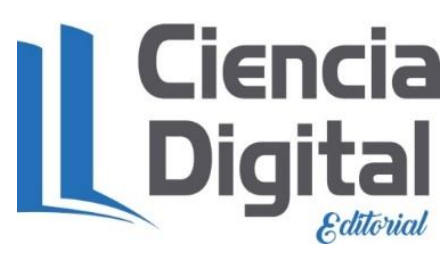

El artículo que se publica es de exclusiva responsabilidad de los autores y no necesariamente reflejan el pensamiento de la Revista Explorador Digital.

El articulo queda en propiedad de la revista y, por tanto, su publicación parcial y/o total en otro medio tiene que ser autorizado por el director o editor de la Revista Explorador Digital.

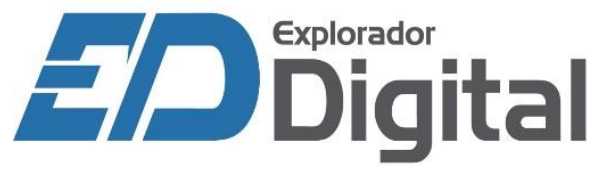

